\title{
石墨烯/纳米减反结构复合透明导电薄膜的研究
}

\author{
韩霜霜，刘莉月，单永奎，杨 帆，李德增 \\ (华东师范大学 化学与分子工程学院, 上海 200241)
}

摘 要: 石墨烯具有较高的透过率及良好的电导率，作为透明导电薄膜具有潜在的应用价值。首先在石英基底上 引入 $\mathrm{SiO}_{2}$ 纳米球阵列结构作为光学减反射层, 使石英基底可见光区光学透过率从 $93.2 \%$ 增加到 $99.0 \%$ 。然后利 用常压化学气相沉积方法，通过基底表面铜颗粒远程催化碳源，直接在减反层上可控制备具有石墨烯/纳米减反 结构的新型复合透明导电薄膜。通过去除 $\mathrm{SiO}_{2}$ 纳米球阵列结构形成反相复制的石墨烯空心球阵列结构, 且生长 时间 $10 \mathrm{~min}$ 时, 对应半高宽约 $40 \mathrm{~cm}^{-1}, I_{2 \mathrm{D}} / I_{\mathrm{G}}=2.31, I_{\mathrm{D}} / I_{\mathrm{G}}=0.77$, 证明在 $\mathrm{SiO}_{2}$ 纳米球阵列减反结构上制备了低缺 陷且连续的全包覆少层石墨烯薄膜。引入 $\mathrm{SiO}_{2}$ 纳米球阵列减反结构后, 其在可见光区光学 $550 \mathrm{~nm}$ 波长处的透过 率平均提高了 $5.5 \%$, 方块电阻相对无减反射层基底平均降低了 $20.09 \%$ 。本研究方法避免了复杂的转移工序, 减 少了对石墨烯的损失与破坏，同时实现了高透光性及高导电性的功能协同，在光伏器件、平板显示等领域展示出 更大的应用前景。

关 键 词: 透明导电薄膜; 石墨烯; 化学气相沉积; 铜颗粒辅助远程催化; 纳米减反结构 中图分类号: TQ174 文献标识码: A

\section{Research of Graphene/Antireflection Nanostructure Composite Transparent Conducting Films}

\author{
HAN Shuang-Shuang, LIU Li-Yue, SHAN Yong-Kui, YANG Fan, LI De-Zeng \\ (School of Chemistry and Molecular Engineering, East China Normal University, Shanghai 200241, China)
}

\begin{abstract}
The graphene film was directly deposited on $\mathrm{SiO}_{2}$ antireflection(AR) structure by remote catalyzation of $\mathrm{Cu}$ nanoparticles using chemical vapor deposition method to fabricate the transparent conducting films with graphene/AR composite structure. Continuous graphene hollow sphere array was obtained after removing $\mathrm{SiO}_{2} \mathrm{AR}$ structure, and the peak intensity ratios of $I_{2 \mathrm{D}} / I_{\mathrm{G}}$ and $I_{\mathrm{D}} / I_{\mathrm{G}}$ and the full-width at half-height maximum (FWHM) of the $2 \mathrm{D}$ peak in the Raman spectrum of the graphene grew in $10 \mathrm{~min}$ were $2.31,0.77$ and about $40 \mathrm{~cm}^{-1}$, respectively, which demonstrated that the continuous and low-defect few layer graphene was grown on the surface of $\mathrm{SiO}_{2} \mathrm{AR}$ structure. By introducing the $\mathrm{SiO}_{2}$ AR structure, transmittance of the film increases by $5.5 \%$ at $550 \mathrm{~nm}$ and the sheet resistance decreases by $20.09 \%$ on the average. Data from this study suggest that this film can avoid complex transfer process, decrease damage, and on the meantime realize high transparency and high conductivity performance, showing obvious applicable prospects in the field of photovoltaic devices, flat panel display and so on.
\end{abstract}

Key words: transparent conducting films; graphene; $\mathrm{CVD}$; remote catalyzation of $\mathrm{Cu}$ nanoparticles; $\mathrm{SiO}_{2}$ antireflection nanostructure 
透明导电薄膜以其接近金属的导电性和可见光 区的高透光性, 被广泛应用在太阳能电池和显示屏 等方面 ${ }^{[1]}$ 。目前广泛使用的氧化锡铟(ITO)因其原料 稀少、化学性质不稳定及缺乏柔韧性等缺点, 应用 日益受限 ${ }^{[2]}$ 。石墨烯具有超高的载流子迁移率(超过 $\left.20,000 \mathrm{~cm}^{2} / \mathrm{Vs}\right)^{[3]}$, 高的光学透过率 $(97.7 \%)^{[4]}$, 优良 的热力学特性 $(5000 \mathrm{~W} /(\mathrm{m} \cdot \mathrm{K}))^{[5-6]}$, 并且具有优异的 柔韧性, 自从被发现以来一直是科学研究的热点, 它作为透明电极的潜力也非常巨大 ${ }^{[7]}$ 。通过进一步 提高基底的透光率, 并结合石墨烯, 制备出兼具高 透光性及高导电性的复合透明导电薄膜, 将会具有 更大的应用前景。

化学气相沉积法(CVD)是目前制备大尺寸石墨 烯薄膜最具工业化前景的方法 ${ }^{[8]}$ 。但该方法通常以 金属铜、镍等为衬底, 为得到单独石墨烯需要复杂 的转移工序, 且在转移过程中不可避免地会对石墨 烯造成破坏 ${ }^{[9]}$ 。因此, 在绝缘祄底上直接生长石墨烯 成为一种发展趋势。研究人员不断尝试在绝缘衬底 上(如 $\mathrm{MgO}^{[10]} 、 \mathrm{Al}_{2} \mathrm{O}_{3}{ }^{[11]} 、 \mathrm{SiO}_{2}{ }^{[12]} 、 \mathrm{SiN}^{[13]}$ 等)生成石 墨烯薄膜, 但由于绝缘祄底与石墨烯晶格不匹配, 生长的石墨烯片层较小, 晶界较多, 降低了石墨烯 的性能。另外, 在绝缘祄底上直接沉积较高质量的 石墨烯, 生长温度通常要高于 $1200^{\circ} \mathrm{C}^{[14]}$ 。本研究首先 在石墨烯薄膜与石英基底之间引入光学减反射结构, 利用光学上的薄膜干涉相消现象, 增加对光透过率 的调控。 $\mathrm{SiO}_{2}$ 光学减反射结构因其结构易控 ${ }^{[15-16]}$ 、 折射率可调节 ${ }^{[17]}$ 、材料易获取而得到广泛应用。本 工作利用溶胶一凝胶法制备出 $\mathrm{SiO}_{2}$ 纳米球溶胶, 再 通过自组装的方法制备 $\mathrm{SiO}_{2}$ 纳米球阵列减反结构, 然后利用浸渍在减反射层表面的乙酸铜高温分解的 铜颗粒远程催化碳源分解, 直接在减反射层表面生 长连续且低缺陷的石墨烯, 得到具有石墨烯/纳米减 反射层/基底结构的新型复合透明导电薄膜。

\section{1 实验方法}

\section{1 石墨烯/石英基底透明导电薄膜的制备 (GE/quartz)}

将清洗干净的石英基底在 $0.1 \mathrm{~mol} / \mathrm{L}$ 乙酸铜的 乙醇溶液中浸泡 $10 \mathrm{~min}$, 然后置于 CVD 管式炉中央, 排空气后 $40 \mathrm{~min}$ 将管式炉升温到反应温度 $1000^{\circ} \mathrm{C}$, 保温 $30 \mathrm{~min}$ 后, $\mathrm{CH}_{4} 、 \mathrm{H}_{2} 、 \mathrm{Ar}$ 流量分别调整为 15 、 5 及 $230 \mathrm{sccm}$, 利用乙酸铜高温分解生成的铜颗粒 远程催化甲烷分解, 在祄底上生长石墨烯。反应结 束后关闭甲烷, 自然冷却至室温, 得到石墨烯/石英
基底结构的透明导电薄膜(GE/quartz)。

\section{$1.2 \mathrm{SiO}_{2}$ 纳米球阵列减反结构的制备 $\left(\mathrm{SiO}_{2}\right.$ NSs/quartz)}

先利用改进后的 Stober 法 ${ }^{[18]}$ 制备单分散 $\mathrm{SiO}_{2}$ 纳米球。具体方法如下: 将摩尔比为 0.1: 40:0.5 的 正硅酸乙酯、去离子水及浓氨水溶于无水乙醇, 在 $45^{\circ} \mathrm{C}$ 恒温水浴中搅拌反应 $3 \mathrm{~h}$ 得到 $\mathrm{SiO}_{2}$ 纳米球溶 胶。再采用浸涂层状自组装方法制备 $\mathrm{SiO}_{2}$ 纳米球阵 列减反结构, 具体过程如下: (1)清洗干净的石英片 先用 $50^{\circ} \mathrm{C}$ 、浓度 $8 \%$ 的 $\mathrm{NaOH}$ 溶液处理 $2 \mathrm{~min}$; (2)通 过提拉镀膜技术交替浸入 $2 \mathrm{wt} \%$ 的聚电解质 $\operatorname{PDDA}$ (聚电解质聚二烯丙基二甲基胺盐酸盐)和 $\operatorname{PSS}$ (聚苯乙烯磺酸钠)溶液中各 $2 \mathrm{~min}$, 共 5 次, 每次 取出用蒸馏水清洗, 得到均匀的(PDDA/PSS) $)_{5} \mathrm{PDDA}$ 涂层; (3)最后在 $\mathrm{SiO}_{2}$ 纳米球溶胶中浸渍 $30 \mathrm{~min}$, 浸 渍后以 $2500 \mu \mathrm{m} / \mathrm{s}$ 的速度匀速提拉得到带有 $\mathrm{SiO}_{2}$ 纳 米球减反结构的基底 $\left(\mathrm{SiO}_{2} \mathrm{NSs} \mathrm{AR} /\right.$ quartz $) 。 \mathrm{SiO}_{2}$ 纳米球阵列减反结构的减反性能的具体优化见辅助 支持材料(图 S1-S3)。

\section{3 石墨烯/纳米减反结构/基底结构复合透明

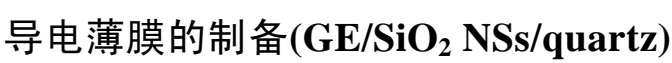

制备好的 $\mathrm{SiO}_{2} \mathrm{NSs} \mathrm{AR} /$ quartz 在 $0.1 \mathrm{~mol} / \mathrm{L}$ 乙酸 铜的乙醇溶液中浸泡 $10 \mathrm{~min}$, 按 1.2 步骤在 $1000^{\circ} \mathrm{C}$, $\mathrm{CH}_{4} 、 \mathrm{H}_{2} 、 \mathrm{Ar}$ 流量分别为 $15 、 5$ 及 $230 \mathrm{sccm}$ 的条件 下, 利用乙酸铜高温分解生成的铜颗粒远程催化甲 烷分解, 在 $\mathrm{SiO}_{2}$ 纳米球阵列减反结构上生长石墨 烯, 得到具有石墨烯/纳米减反结构/基底结构的复 合透明导电薄膜 $\left(\mathrm{GE} / \mathrm{SiO}_{2} \mathrm{NSs}\right.$ AR /quartz)。

\section{4 测试与表征}

通过用场发射扫描电子显微镜(FESEM, Hitachi S-4800)观察样品的表面形貌, 并用 EDS 进行元素 分析; 通过透射电子显微镜(TEM, JEOL 2100F)和 高分辨透射电镜观察样品结构。为了得到单独的石 墨烯, 用氢氟酸(HF) 溶液腐蚀掉 $\mathrm{SiO}_{2}$ 纳米球及石英 基底, 再将石墨烯清洗后转移到铜网上进行 TEM 表征; 利用拉曼光谱仪(Raman, Thermo DXR Raman microscope, 激发波长为 $532 \mathrm{~nm}$ )测定石墨烯的层 数、晶体结构等信息; 采用紫外可见光谱(UV-8000, Metash) 测试薄膜在可见光区的光学透过率; 利用 四探针法 (RTS-7)测定透明导电薄膜方块电阻。

\section{2 结果与讨论}

为了得到 $\mathrm{GE} / \mathrm{SiO}_{2} \mathrm{NSs} \mathrm{AR} /$ quartz 复合透明导电 薄膜, 利用铜颗粒远程催化, 在 $\mathrm{SiO}_{2} \mathrm{NSs} \mathrm{AR} /$ quartz 
减反层上直接生长石墨烯。通过控制生长时间可控 制备出了不同石墨烯层数的复合透明导电薄膜。

为了表征样品的形貌和结构, 在扫描电镜下观 察生长时间为 $20 \mathrm{~min}$ 的 $\mathrm{GE} / \mathrm{SiO}_{2} \mathrm{NSs} \mathrm{AR}$ /quartz 样 品, 结果如图 1 所示。图 1 中的 $(a, b)$ 分别对应为未 生长石墨烯的 $\mathrm{SiO}_{2} \mathrm{NSs}$ AR /quartz 和生长石墨烯的 $\mathrm{GE} / \mathrm{SiO}_{2} \mathrm{NSs} \mathrm{AR} /$ quartz 样品的 SEM 形貌, 从图 1(a) 可以看出制备的 $\mathrm{SiO}_{2}$ 纳米球颗粒大小均匀, 呈单层 单分散状态。从图 1(b)可以看出经过高温生长石墨 烯后, $\mathrm{SiO}_{2}$ 纳米球形貌保持完好, 且大范围内无残 留的铜纳米粒子。由于祄度原因从图 1(b)中较难看 出石墨烯, 可以从图 1(b)的区域放大图中看出二氧 化硅纳米球减反膜已被石墨烯所覆盖。从 SEM 照片 中选取残留有铜纳米粒子的区域进行 EDS 表征如图 1(c), 白色区域圈出的亮点即为残留的铜纳米粒子, 而灰色祄度的颗粒均为 $\mathrm{SiO}_{2}$ 纳米球, 可以看出绝大 部分作为辅助催化的铜纳米粒子在升降温及高温生 长过程中变成铜蒸汽催化甲烷分解后随气流带走, 只有极少数铜纳米粒子留在基底上, 这也是复合薄 膜能保持高透光度的一个重要原因。

通过透射电子显微镜和高分辨透射电镜观察样 品结构信息, 图 2 (a)表明该法能够在 $\mathrm{SiO}_{2}$ 纳米球阵
列减反结构上有效地生长连续的石墨烯薄膜，且石 墨烯能较紧密地全包覆纳米球，而不是简单地铺在 $\mathrm{SiO}_{2}$ 纳米球表面。图 2(b,c)所示为去除 $\mathrm{SiO}_{2} \mathrm{NSs}$ 后 石墨烯空心球结构的 TEM 照片, 可见除去硅球后 石墨烯能复制并保留独特的石墨烯空心球结构。

图 3 和表 1 分别为 $1000^{\circ} \mathrm{C}$ 生长不同时间得到的 $\mathrm{GE} / \mathrm{SiO}_{2} \mathrm{NSs}$ AR film/quartz 复合薄膜样品的拉曼光 谱图及其数据分析。生长时间 $10 \mathrm{~min}$ 时, $2 \mathrm{D}$ 峰位于 $2697 \mathrm{~cm}^{-1}$, 对应半高宽约 $40 \mathrm{~cm}^{-1}$, 且 $I_{2 \mathrm{D}} / I_{\mathrm{G}}=2.31$, $I_{\mathrm{D}} / I_{\mathrm{G}}=0.77$, 可见控制生长时间为 $10 \mathrm{~min}$ 时, 可在 $\mathrm{SiO}_{2}$ 纳米球减反膜上制备出低缺陷的单层石墨烯。 生长时间从 $10 \mathrm{~min}$ 增加到 $25 \mathrm{~min}, I_{\mathrm{D}} / I_{\mathrm{G}}$ 比值从 0.77 依次降为 $0.76 、 0.64 、 0.60, I_{2 \mathrm{D}} / I_{\mathrm{G}}$ 比值从 2.31 依次 减小到 $0.95 、 0.84 、 0.49$, 说明随着生长时间的延长, 石墨烯薄膜变厚，尺寸变大缺陷减少，层数随时间 可控地从单层变为多层。根据石墨烯尺寸公式: $L_{a}=$ $\left(2.4 \times 10^{-10}\right) \lambda^{4}\left(I_{\mathrm{G}} / I_{\mathrm{D}}\right)$, 可计算出石墨烯尺寸从 $25 \mathrm{~nm}$ 增加到 $32 \mathrm{~nm}$ 。根据文献[19]报导含有缺陷的石墨烯, 在 $1620 \mathrm{~cm}^{-1}$ 附近还会出现 $\mathrm{D}^{\prime}$ 峰。D 峰和 $\mathrm{D}^{\prime}$ 峰分别 产生于谷间和谷内散射过程, $I_{\mathrm{D}} / I_{\mathrm{D}}$ 的强度比可以对 石墨烯缺陷类型进行判断。研究表明, 对于 $\mathrm{sp}^{3}$ 杂化 产生的缺陷, $I_{\mathrm{D}} / I_{\mathrm{D}^{\prime}}$ 约为 13 ; 空位类型的缺陷, $I_{\mathrm{D}} / I_{\mathrm{D}^{\prime}}$
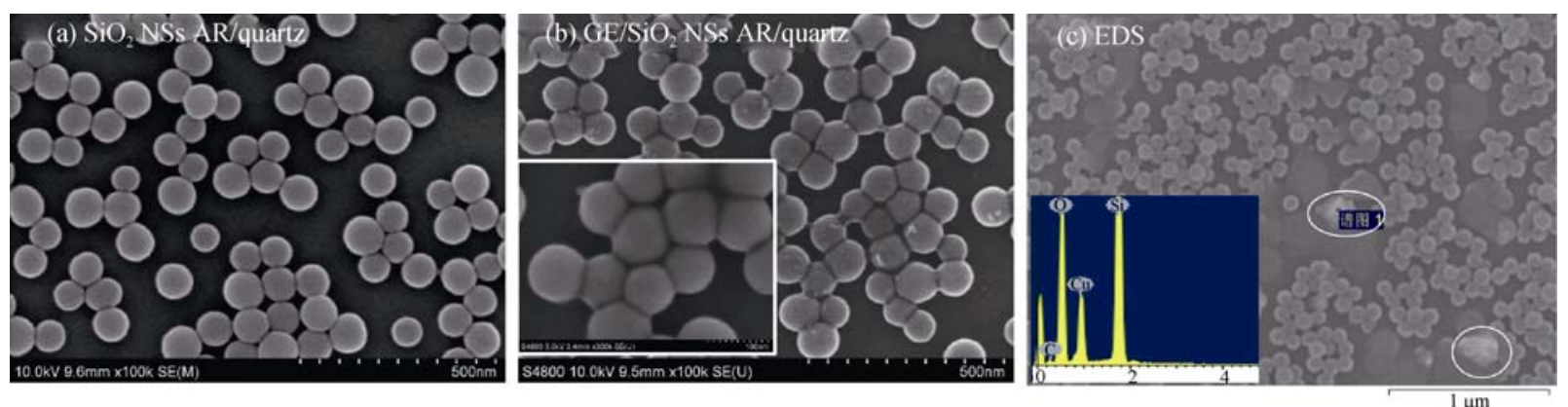

图 1 未生长石墨烯的 $\mathrm{SiO}_{2} \mathrm{NSs}$ AR /quartz 的 SEM 照片(a), 生长石墨烯的 GE/SiO 2 NSs AR film/quartz 的 SEM 照片及其高倍放 大照片(b), 残留在基底上的铜纳米粒子的 EDS 表征(c)

Fig. 1 SEM images of monodisperse $\mathrm{SiO}_{2}$ NSs structure before (a) and after (b) growth of graphene and the magnification image of $\mathrm{GE} / \mathrm{SiO}_{2} \mathrm{NSs}(\mathrm{c})$ with EDS characterization of the $\mathrm{Cu}$ NPs left on the substrate in (c)
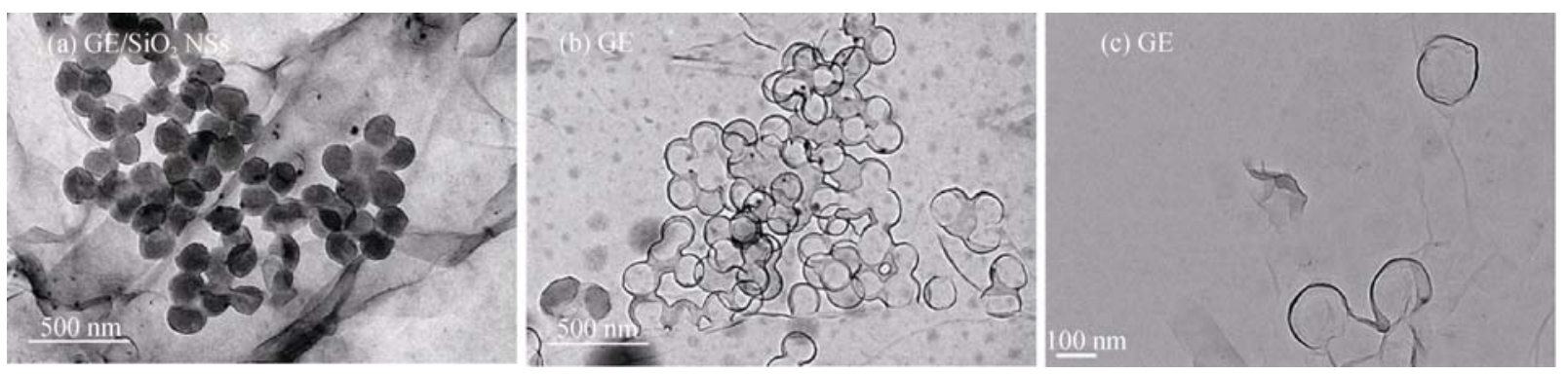

图 2 (a) GE/ $\mathrm{SiO}_{2} \mathrm{NSs}$ 和(b)去除 $\mathrm{SiO}_{2} \mathrm{NSs}$ 后的石墨烯空心球结构的 TEM 照片, (c)石墨烯空心球结构的高倍 TEM 照片

Fig. 2 TEM images of GE/SiO $\mathrm{SSs}_{2}$ /quartz (a) and hollow graphene NSs (b) by removing $\mathrm{SiO}_{2} \mathrm{NSs}$, high magnification TEM image of hollow graphene NSs (c) 
表 1 不同生长时间下生长的石墨烯的拉曼光谱数据分析

Table 1 Raman spectra analysis of graphene grown for different time

\begin{tabular}{cccccc}
\hline \multirow{2}{*}{ Growth time } & \multicolumn{2}{c}{$2 \mathrm{D}$ band } & & & \\
\cline { 2 - 3 } & \multicolumn{2}{c}{ Position } & $\mathrm{cm}_{\mathrm{D}} / I_{\mathrm{G}}$ & $I_{2 \mathrm{D}} / I_{\mathrm{G}}$ & $L_{\mathrm{a}} / \mathrm{nm}$ \\
\cline { 2 - 3 } & 2697.60 & $\mathrm{~cm}^{-1}$ & & & \\
\hline $10 \mathrm{~min}$ & 2691.69 & 40 & 0.77 & 2.31 & 24.9 \\
$15 \mathrm{~min}$ & 2695.55 & 56 & 0.76 & 0.95 & 25.3 \\
$20 \mathrm{~min}$ & 2690.72 & 55 & 0.64 & 0.84 & 30.0 \\
$25 \mathrm{~min}$ & & 56 & 0.60 & 0.49 & 32.0 \\
\hline
\end{tabular}

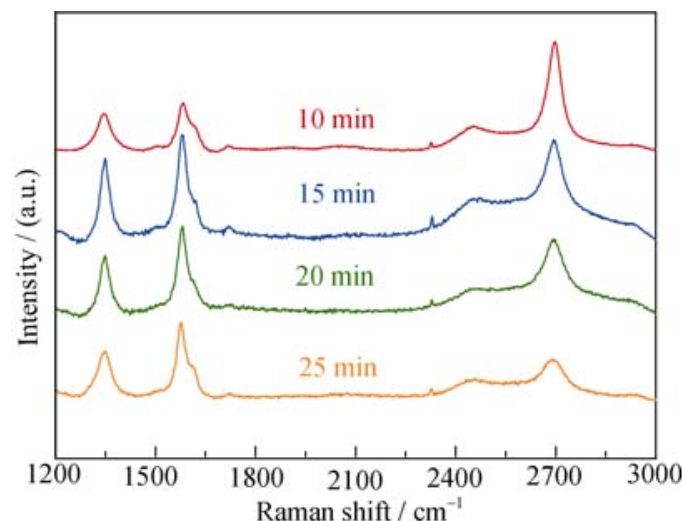

图 $31000^{\circ} \mathrm{C}$ 生长不同时间得到的 $\mathrm{GE} / \mathrm{SiO}_{2} \mathrm{NSs}$ /quartz 样品 的拉曼光谱图

Fig. 3 Raman spectra of $\mathrm{GE} / \mathrm{SiO}_{2} \mathrm{NSs} /$ quartz grown at $1000^{\circ} \mathrm{C}$ for different time

比值约为 7 ; 而石墨烯边缘的缺陷, $I_{\mathrm{D}} / I_{\mathrm{D}^{\prime}}$ 比值约为 3.5 。经过计算, 本实验制备的石墨烯 $I_{\mathrm{D}} / I_{\mathrm{D}}$ 缺陷比值 小于 3.5 , 所以主要为边缘缺陷。而缺陷密度和 $I_{\mathrm{D}} / I_{\mathrm{G}}$ 的强度比之间存在如下关系: $r_{\mathrm{D}}\left(\mathrm{cm}^{-2}\right)=(7.3 \pm 2.2) \times$ $10^{9} E_{\mathrm{L}}{ }^{4}\left(I_{\mathrm{D}} / I_{\mathrm{G}}\right)$, 生长时间从 $10 \mathrm{~min}$ 延长到 $25 \mathrm{~min}$, 经 计算缺陷密度从 $1.15 \times 10^{4} \mathrm{~cm}^{-2}$ 减小到 $9 \times 10^{3} \mathrm{~cm}^{-2}$ 。 虽然比金属箔基底上生长的石墨烯缺陷多, 但明显 比无催化剂直接在绝缘沉底上沉积的石墨烯缺陷少, 直接高温沉积方法制备出的石墨烯的 $\mathrm{D}$ 峰与 $\mathrm{G}$ 峰的 比值会大于 1 。

在表征石墨烯/纳米减反结构复合透明导电薄 膜光学性质之前, 首先对所制备 $\mathrm{SiO}_{2}$ 纳米球阵列减 反结构的减反效果进行了表征, 从图 4 可以看出增 加减反结构后增透效果明显, 透光度有了大幅度提 高。550 nm 处, 玻璃基底透光度从 $91.2 \%$ 提高到 $99.3 \%$, 提高了 $8.1 \%$; 石英基底透光度从 $93.2 \%$ 提高 到 $99.0 \%$, 提高了 $5.8 \%$, 为实现高透高导 $\mathrm{GE} / \mathrm{SiO}_{2}$ $\mathrm{NSs}$ AR/quartz 复合透明导电薄膜提供了高透光的 基础。

对不同生长时间制备的 $\mathrm{GE} / \mathrm{SiO}_{2} \mathrm{NSs} /$ quartz 复 合透明导电薄膜的光学透过率和方块电阻进行了测

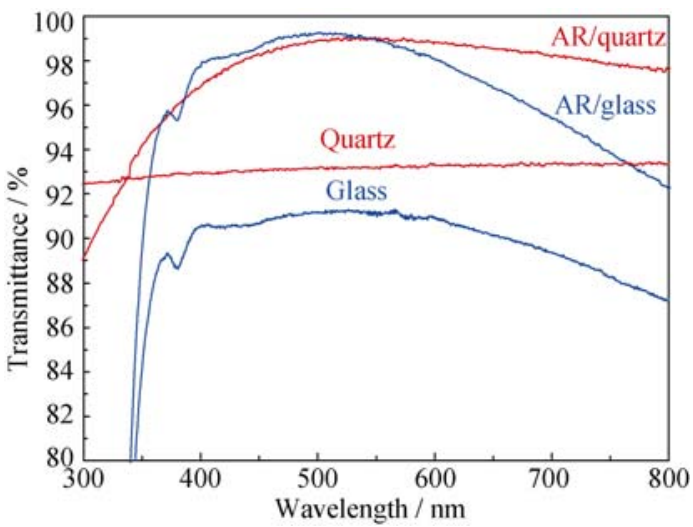

图 4 有无 $\mathrm{SiO}_{2}$ 纳米球阵列减反结构的透光度对比图

Fig. 4 Transmission spectra of the substrate coated with and without $\mathrm{SiO}_{2} \mathrm{NSs}$ AR nanostructure

试, 结果如图 5 所示。从图 5 及其表 2 中可以看出, 随着生长时间延长, $\mathrm{GE} / \mathrm{SiO}_{2} \mathrm{NSs} /$ quartz 复合透明 导电薄膜的透过率减小，从 $96.3 \%$ 降到 $83 \%$ 。由于 石墨烯片层尺寸增大，层数变多，石墨烯片间的晶 界减少，减少了因片层间的晶格错位引起的载流子 散射，所以随着生长时间的延长，载流子迁移率增 大, 方块电阻从 $20.5 \mathrm{k} \Omega / \square$ 降到 $0.9 \mathrm{k} \Omega /$, 导电性增 强。通过与 GE/quartz 实验数据对比结果表 2 可以 发现, $\mathrm{GE} / \mathrm{SiO}_{2} \mathrm{NSs} /$ quartz 复合透明导电薄膜的透 过率比未加 $\mathrm{SiO}_{2}$ 纳米球减反结构的 $\mathrm{GE} /$ quartz 有了 显著提高。复合薄膜在 $550 \mathrm{~nm}$ 处的透光率相对无减 反射层基底平均改变了 $5.5 \%$, 平均提高比率为 $6.34 \%$, 其方块电阻相对无减反射层基底平均降低 了 $20.09 \%$, 保持较好的透光率和导电性。

\section{3 结论}

采用溶胶一凝胶法和自组装技术制备出高减反 效果的 $\mathrm{SiO}_{2}$ 纳米球阵列减反结构, 所制备的 $\mathrm{SiO}_{2}$ 纳米球阵列减反结构使石英基底透过率从 93.2\%增 加到 $99.0 \%$ ，提高了 $5.8 \%$ 。并利用铜颗粒远程催化 法成功在 $\mathrm{SiO}_{2}$ 纳米球阵列减反结构上直接生长了 

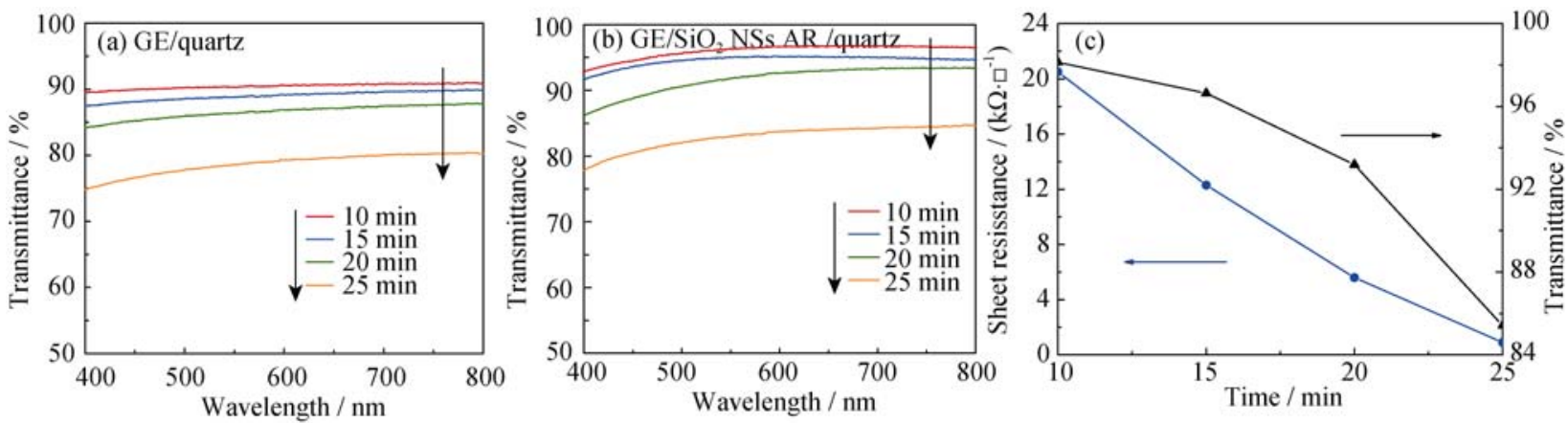

图 5 (a)不同生长时间制备的 $\mathrm{GE} /$ quartz 的光学透过率谱图, (b) GE/ $\mathrm{SiO}_{2} \mathrm{NSs}$ AR /quartz 的光学透过率谱图, (c)550 nm 处透过率 和方块电阻对生长时间的折线图

Fig. 5 Optical transmittance spectra of the $\mathrm{GE} / \mathrm{SiO}_{2} \mathrm{NSs} \mathrm{AR} /$ quartz (a), transmittance (at $550 \mathrm{~nm}$ ) (b) and sheet resistance as a function of growth time(c)

表 2 样品的透过率和方块电阻的数据

Table 2 Transmittance and sheet resistance of samples

\begin{tabular}{cccccc}
\hline & Growth time & $10 \mathrm{~min}$ & $15 \mathrm{~min}$ & $20 \mathrm{~min}$ & $25 \mathrm{~min}$ \\
\hline \multirow{3}{*}{ Transmittance/\% } & $\mathrm{GE} /$ quartz & 90.30 & 88.90 & 86.40 & 78.60 \\
& $\mathrm{GE} / \mathrm{SiO}_{2} \mathrm{NS}$ AR film/quartz & 96.30 & 95.00 & 91.80 & 83.00 \\
& Increment/\% & 6.640 & 6.86 & 6.25 & 5.60 \\
Sheet resistance $/\left(\mathrm{k} \Omega \cdot \cdot^{-1}\right)$ & $\mathrm{GE} /$ quartz & -- & 16.30 & 6.07 & 1.25 \\
& $\mathrm{GE} / \mathrm{SiO}_{2} \mathrm{NS} \mathrm{AR} \mathrm{film/quartz}$ & 20.50 & 12.30 & 5.60 & 0.90 \\
& Decrease/\% & & 24.54 & 7.74 & 28.00 \\
\hline
\end{tabular}

连续低缺陷层数可控的石墨烯, 制备出 $\mathrm{GE} / \mathrm{SiO}_{2}$ NSs AR /quartz 复合透明导电薄膜。所沉积的石墨 烯能较好复制纳米球阵列结构, 简单制备出全包 覆的 $\mathrm{GE} / \mathrm{SiO}_{2} \mathrm{NSs}$ 复合材料, 这是液相法和用金属 祄底 CVD 法再转移难以做到的。并且引入 $\mathrm{SiO}_{2}$ 纳 米球阵列减反结构后, $\mathrm{GE} / \mathrm{SiO}_{2} \mathrm{NSs} \mathrm{AR} / \mathrm{quartz}$ 复 合透明导电薄膜的透过率较 $\mathrm{GE} /$ quartz 平均提高了 $5.5 \%$, 平均提高比率为 $6.34 \%$, 其方块电阻相对无 减反射层基底平均降低了 $20.09 \%$, 保持较好的透 光率和导电性。研究制备的 $\mathrm{GE} / \mathrm{SiO}_{2} \mathrm{NSs} \mathrm{AR} /$ quartz 复合透明导电薄膜的透光性高于传统的透明导电 膜，作为透明导电薄膜的应用潜力巨大，制备的材 料可用作太阳能电池窗口电极材料应用到光伏、导 电等诸多领域, 能极大地扩大透明导电薄膜的应 用范围。

\section{参考文献:}

[1] WEISS N O, ZHOU H, LIAO L, et al. Graphene: an emerging electronic material. Adv. Mater., 2012, 24(43): 5782-5825.

[2] GOMEZ D A L, ZHANG Y, SCHLENKER C W, et al. Continuous, highly flexible, and transparent graphene films by chemical vapor deposition for organic photovoltaics. ACS Nano, 2010, 4(5): 2865.

[3] GEIM A K, NOVOSELOV K S. The rise of graphene. Nature Ma- terials, 2007, 6(3): 183-191.

[4] NAIR R R, BLAKE P, GRIGORENKO A N, et al. Fine structure constant defines visual transparency of graphene. Science, 2008, 320(5881): 1308

[5] BOLOTIN K I, SIKES K J, JIANG Z, et al. Ultrahigh electron mobility in suspended graphene. Solid State Commun., 2008, 146(9): 351-353.

[6] KIM K S, ZHAO Y, JANG H, et al. Large-scale pattern growth of graphene films for stretchable transparent electrodes. Nature, 2009, 457(7230): 706-710.

[7] RAO C N R, SOOD A K, SUBRAHMANYAM K S. The new two-dimensional nanomaterial. Angew. Chemie, 2009, 48(42): $7752-7777$.

[8] CAO H, YU Q, COLBY R, et al. Large-scale graphitic thin films synthesized on $\mathrm{Ni}$ and transferred to insulators: structural and electronic properties. J. Appl. Phys., 2010, 107(4): 044310.

[9] BHAVIRIPUDI S, JIA X, DRESSELHAUS M. S, et al. Role of kinetic factors in chemical vapor deposition synthesis of uniform large area graphene using copper catalyst. Nano Lett., 2010, 10(10): $4128-4133$.

[10] GADDAM S, BJELKEVIG C, GE S, et al. Direct graphene growth on MgO: origin of the band gap. J. Phys. Condens. Matter., 2011, 23(7): 072204.

[11] JERNG S, YU D, KIM Y, et al. Nanocrystalline graphite growth on 
sapphire by carbon molecular beam epitaxy. J. Phys. Chem. C, 2011, 115(11): 4491-4494.

[12] BI H, SUN S, HUANG F, et al. Direct growth of few-layer graphene films on $\mathrm{SiO}_{2}$ substrates and their photovoltaic applications. J. Mater. Chem., 2012, 22(2): 411-416.

[13] CHEN J, GUO Y, WEN Y, et al. Two-stage metal-catalyst-free growth of high-quality polycrystalline graphene films on silicon nitride substrates. Adv. Mater., 2013, 25(7): 992-997.

[14] SU C Y, LU A Y, WU C Y, et al. Direct formation of wafer scale graphene thin layers on insulating substrates by chemical vapor deposition. Nano Letters, 2011, 11(9): 3612-3616.

[15] GREEN M A. Third Generation Photovoltaics: Advanced Solar Energy Conversion. Berlin: Springer Press, 2005: 35-38.
[16] LI D Z, WAN D Y, X. ZHU L, et al. Broadband antireflection $\mathrm{TiO}_{2}-\mathrm{SiO}_{2}$ stack coatings with refractive-index-grade structure and their applications to $\mathrm{Cu}(\mathrm{In}, \mathrm{Ga}) \mathrm{Se}_{2}$ solar cells. Solar Energy Materials \& Solar Cells, 2014, 130(7): 505-512.

[17] LI D Z, WU G M, GAO G H, et al. Ultrafast coloring-bleaching performance of nanoporous $\mathrm{WO}_{3}-\mathrm{SiO}_{2}$ gasochromic films doped with Pd catalyst. ACS Applied Materials \& Interfaces, 2011, 3(3): 4573-4579.

[18] LIU B T, YEH W D. Antireflective surface fabricated from colloidal silica nanoparticles. Colloids and Surfaces A: Physicochem. Eng. Aspects, 2010, 356(1/2/3): 145-149.

[19] WU JUAN-XIA, XU HUA, ZHANG JIN. Raman spectroscopy of graphene. Acta Chim. Sinica, 2014, 72(3): 301-318. 


\section{辅助支持材料: $\mathrm{SiO}_{2}$ 纳米球阵列减反结构的减反性能优化}

\section{1. 水硅比对 $\mathrm{SiO}_{2}$ 纳米球阵列减反结构减反性能的影响}

溶胶中水和硅摩尔比分别设定为 $\mathrm{H}_{2} \mathrm{O}: \mathrm{TEOS}=1: 20 / 40 / 60$, 镀膜过程中浸渍 $60 \mathrm{~min}$, 提拉速度 $2000 \mu \mathrm{m} / \mathrm{s}$, 测其在可见光区的透光率, 得出水硅比与 $\mathrm{SiO}_{2}$ 纳米球阵列减反结构透光率之间的变化规律, 结果如图 $\mathrm{S} 1$ 。 从图 $\mathrm{S} 1$ 中可以看出, 随着水硅比的增大, TEOS 水解的速度增大, 生成的 $\mathrm{SiO}_{2}$ 纳米球粒径增大, 从 $\mathrm{SEM}$ 照 片可以看出这一趋势, 也可粗略看出 $n\left(\mathrm{H}_{2} \mathrm{O}\right): n$ (TEOS)分别为 20、40、60 时对应纳米球粒径分别为 $60 \sim 70 \mathrm{~nm}$ 、 90 100 nm、110 120 nm。根据布拉格定律、菲涅耳反射系数理论和有效介质理论研究出的优化最佳减反射 结构参数的公式可知当入射光角度一定时，带隙中心波长位置与胶体纳米球的粒径呈线性递增关系。这从 图 S1(a)透光度图可得到证实，随着水硅比的增大，纳米球的粒径增大，中心波长的位置红移。另外从 SEM 照片中也可以看到随着水硅比的增大, 纳米球的粒径增大, 薄膜空隙率减小, 所以薄膜透光度降低, 实验结 果与理论吻合。为充分利用太阳光, 减少薄膜在可见光区段的反射, 选用水硅比比值为 40 时较为合适。
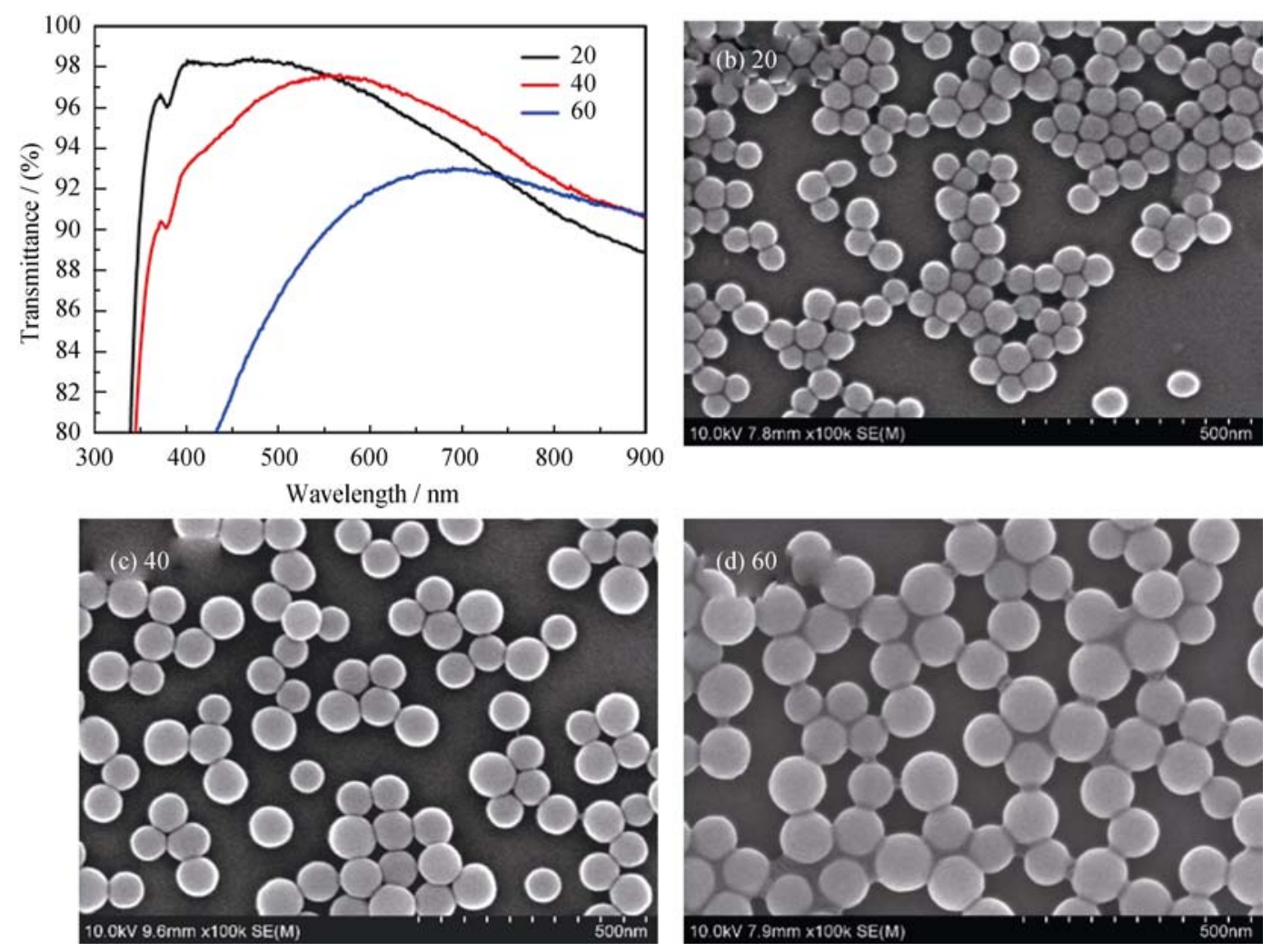

图 $\mathrm{S} 1$ 不同水硅比 $\left(n\left(\mathrm{H}_{2} \mathrm{O}\right): n(\mathrm{TEOS})\right)$ 制备的 $\mathrm{SiO}_{2}$ 纳米球阵列减反结构的透光度图谱(a), 不同水硅比制备的减反结构的 SEM 照片(b d $)$ Fig. S1 (a) Transmission spectra of the $\mathrm{SiO}_{2} \mathrm{NSs}$ AR/quartz with different mole ratios $\left(n\left(\mathrm{H}_{2} \mathrm{O}\right): n(\mathrm{TEOS})\right)$, SEM images of the SiO${ }_{2}$ NSs AR/quartz prepared from different mole ratios $\left(n\left(\mathrm{H}_{2} \mathrm{O}\right): n(\mathrm{TEOS})\right)(\mathrm{b}-\mathrm{d})$

\section{2、浸渍时间对 $\mathrm{SiO}_{2}$ 纳米球阵列减反结构减反性能的影响}

将已均匀组装上 $(\mathrm{PDDA} / \mathrm{PSS})_{5} \mathrm{PDDA}$ 涂层的玻璃片浸入到水硅比为 40 的 $\mathrm{SiO}_{2}$ 纳米球溶胶中, 分别浸渍 不同时间 $10 \mathrm{~s} 、 15 \mathrm{~min} 、 30 \mathrm{~min} 、 45 \mathrm{~min} 、 60 \mathrm{~min}$ 后, 将基片以 $2000 \mu \mathrm{m} / \mathrm{s}$ 的速度平稳地从溶胶中提拉出, 测 其在可见光区的透光率, 得出浸渍时间与 $\mathrm{SiO}_{2}$ 纳米球减反膜透光率之间的变化规律, 结果如图 $\mathrm{S} 2$ 。随着浸 渍时间的延长, 基底上 $\mathrm{SiO}_{2}$ 纳米球的密度增加, 空隙率减小, 由有效折射率和空隙率的关系公式 $n_{\mathrm{c}}=n_{\mathrm{eff}}$ 
$\left[\Phi_{\mathrm{pnp}}{ }^{2}+\left(1-\Phi_{\mathrm{p}}\right) n_{\mathrm{a}}{ }^{2}\right]^{1 / 2}$ 可知导致薄膜有效折射率增大，而薄膜有效折射率与理论匹配最佳的折射率 $n_{\mathrm{c}}=$ $\left(n_{\mathrm{a}} n_{\mathrm{g}}\right)^{1 / 2}=1.23$ 越接近, 减反效果越好, 透光度越高。在浸渍时间为 $30 \mathrm{~min}$ 时, 所得的 $\mathrm{SiO}_{2}$ 纳米球减反性能 最好。

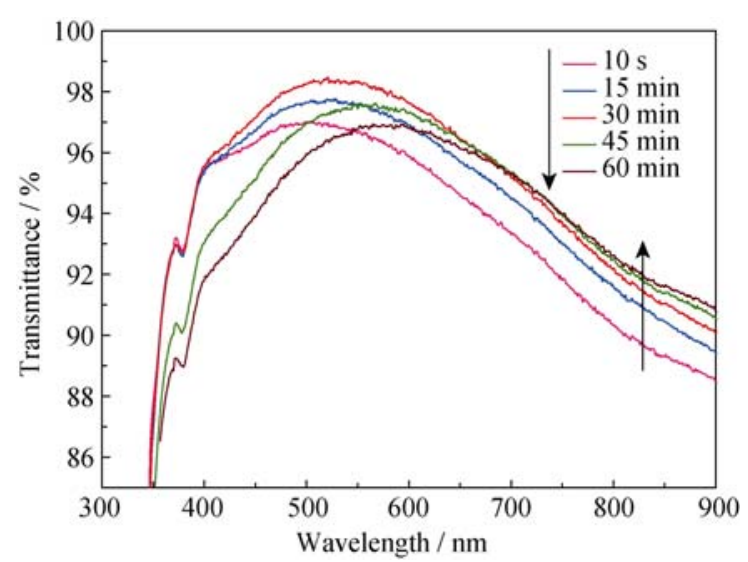

图 S2 不同浸渍时间对 $\mathrm{SiO}_{2}$ 纳米球阵列减反结构透光度的影响

Fig. S2 Influences on $\mathrm{SiO}_{2} \mathrm{NSs}$ AR nanostructure reflection from dipping time

\section{3、提拉速度对 $\mathrm{SiO}_{2}$ 纳米球阵列减反结构减反性能的影响}
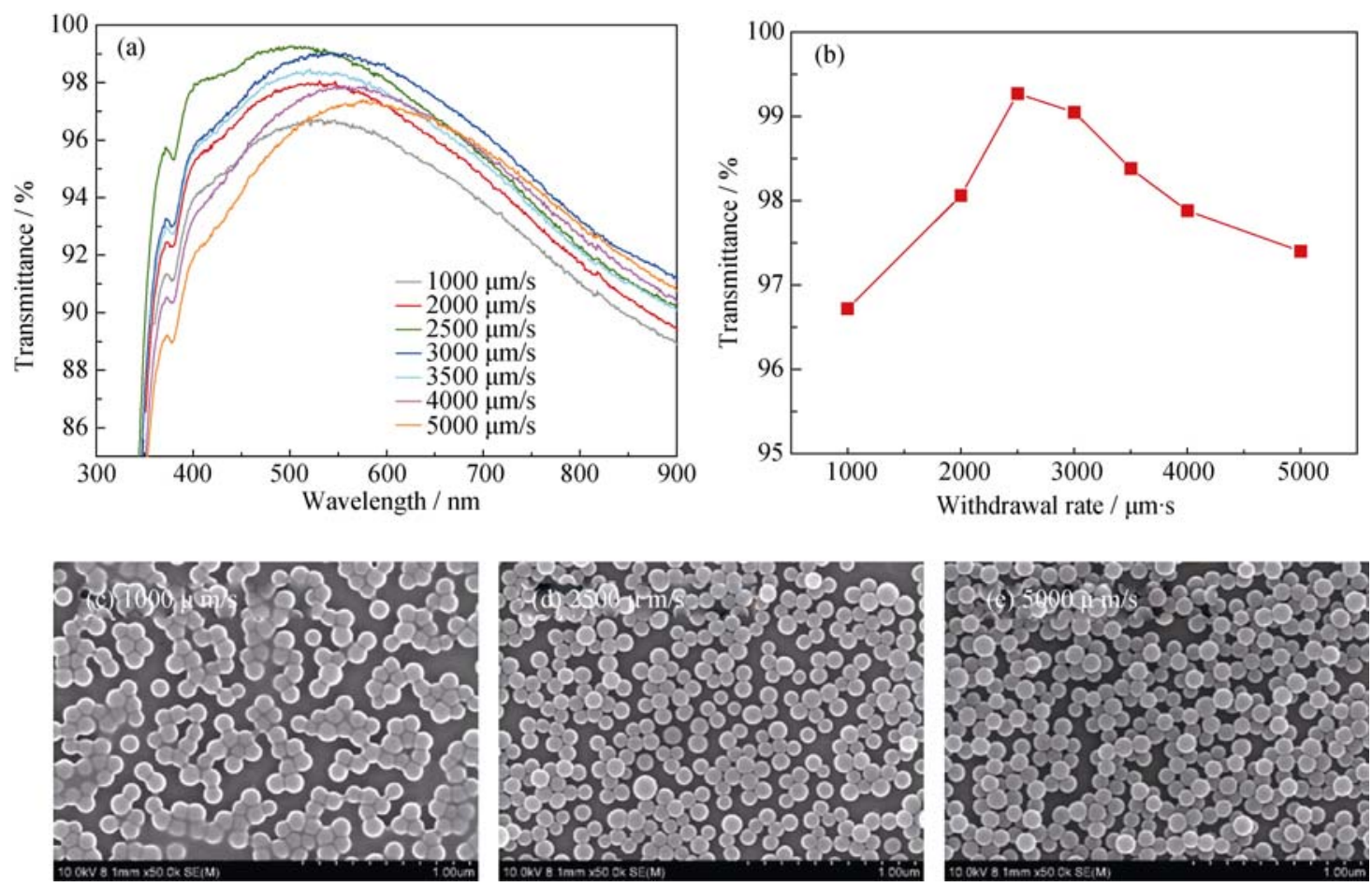

图 $\mathrm{S} 3$ 镀膜提拉速度对 $\mathrm{SiO}_{2}$ 纳米球阵列减反结构减反性能的影响表征

Fig. S3 Relationship between on $\mathrm{SiO}_{2} \mathrm{NSs}$ AR nanostructure reflection from the withdrawal rate

(a) Transmission spectra of the $\mathrm{SiO}_{2}$ NSs AR /quartz with different withdrawal rate, (b) Transmittance of $\mathrm{SiO}_{2} \mathrm{NSs}$ AR /quartz as a function of withdrawal rate, and (c-e) SEM images of the $\mathrm{SiO}_{2} \mathrm{NSs}$ AR /quartz prepared with different withdrawal rates

从图 S3 (a, b)中看出提拉速度从 $1000 \mu \mathrm{m} / \mathrm{s}$ 增加到 $5000 \mu \mathrm{m} / \mathrm{s}$ 时, $\mathrm{SiO}_{2}$ 纳米球减反性能先增加后减小, 在 $2500 \mu \mathrm{m} / \mathrm{s}$ 时达到最大透过率 99.27\%。随着提拉速度的增加，薄膜空隙率减小如 SEM 图(c, d) 所示，当提拉 速度为 $2500 \mu \mathrm{m} / \mathrm{s}$ 时, 薄膜空隙率较合适, 所以减反性能最好。而当速度继续增大到如 $5000 \mu \mathrm{m} / \mathrm{s}$ 时, 薄膜 会出现不均匀多层的情况, 如图 S3(e)所示, 导致透光度显著下降。 